\title{
Thank You to Our Peer Reviewers
}

\section{“Top 50 Reviewers" Acknowledgment}

The $C O R R^{\circledR}$ Board of Trustees would like to acknowledge our Top 50 Reviewers for their excellent work. This group consists of the top 50 reviewers whose average review-quality score ranked the highest, who reviewed four or more times between September 2016 and August 2017, and who are not currently on our Editorial Board. We congratulate our Top 50 Reviewers for their success, we recognize them for their vision, and we thank them for their contributions to the journal and to orthopaedic science.

\begin{tabular}{|c|c|}
\hline Albert Aboulafia & Baltimore, $M D$ \\
\hline Jaimo Ahn & Philadelphia, PA \\
\hline Tessa Balach & Chicago, $I L$ \\
\hline Nikolaos Bardakos & Voula, Greece \\
\hline Thomas Bauer & New York, $N Y$ \\
\hline Wayne Berberian & Maywood, $N J$ \\
\hline Jefferson Brand & Alexandria, $M N$ \\
\hline Darrel Brodke & Salt Lake City, UT \\
\hline Michaël Bus & Leiden, Netherlands \\
\hline Hongsen Chiang & Taipei, Taiwan \\
\hline Timothy Damron & Syracuse, $N Y$ \\
\hline James Dennis & Houston, TX \\
\hline Lawrence Dorr & Los Angeles, $C A$ \\
\hline John Elias & Akron, $\mathrm{OH}$ \\
\hline Peter Fabricant & New York, $N Y$ \\
\hline Brett Freedman & Bethesda, MD \\
\hline Gary Friedlaender & New Haven, $C T$ \\
\hline Freddie Fu & Pittsburgh, PA \\
\hline Kevin Garvin & Omaha, NE \\
\hline Jonathan Godin & Durham, NC \\
\hline Michael Grecula & Houston, TX \\
\hline Anthony Griffin & Toronto, Canada \\
\hline Konrad Gruson & Bronx, $N Y$ \\
\hline Kenneth Gundle & Portland, OR \\
\hline James Hayden & Portland, $O R$ \\
\hline Jason Ho & Cleveland, $\mathrm{OH}$ \\
\hline Robert Hopper & Alexandria, $V A$ \\
\hline Stein Janssen & Amsterdam, Netherlands \\
\hline Bernhard Jost & St. Gallen, Switzerland \\
\hline Michael Joyce & Cleveland, $\mathrm{OH}$ \\
\hline David King & Milwaukee, WI \\
\hline Ken Kuo & Taipei, Taiwan \\
\hline Gwo-Chin Lee & Philadelphia, PA \\
\hline Yuo-yu Lee & New York, $N Y$ \\
\hline
\end{tabular}




$\begin{array}{ll}\text { Thoralf Liebs } & \text { Bern, Switzerland } \\ \text { J. Lawrence Marsh } & \text { Iowa City, IA } \\ \text { Sergio Mendoza-Lattes } & \text { Durham, NC } \\ \text { Richard Nicholas } & \text { Little Rock, AR } \\ \text { Kevin Plancher } & \text { New York, NY } \\ \text { Hollis Potter } & \text { New York, NY } \\ \text { Ola Rolfson } & \text { Gothenburg, Sweden } \\ \text { Keun Jung Ryu } & \text { Gwangmyeong-si, } \\ & \text { Republic of Korea } \\ \text { Andrew Schoenfeld } & \text { Boston, MA } \\ \text { Herbert Schwartz } & \text { Nashville, TN } \\ \text { Gene Siegal } & \text { Birmingham, AL } \\ \text { H. Thomas Temple } & \text { Fort Lauderdale, FL } \\ \text { Kimberly Templeton } & \text { Kansas City, KS } \\ \text { Ana-Maria Vranceanu } & \text { Boston, MA } \\ \text { Emily Jane Woo } & \text { Rockville, } M D \\ \text { Timothy Wright } & \text { Gainesville, FL }\end{array}$

\section{CORR $^{\circledR}$ Thanks its Peer Reviewers}

The $C O R R^{\circledR}$ Board of Trustees thanks the following reviewers for their evaluations of manuscripts from September 2016 through August 2017. The excellence of $C O R R^{\circledR}$ depends on their substantial efforts.

Arild Aamodt
Megan Aanstoos
Joseph Abboud
Ayesha Abdeen
Matthew Abdel
William Abdu
Yousef Abu-Amer
Yvonne Achermann
Philippe Adam
Andrew Adamczyk
Brian Adams
Judith Adams
Samuel Adams
Gregory Adamson
Roberto Adani
Allan Adkin
Max Aebi
Saverio Affatato
Animesh Agarwal
Manish Agarwal
Jose Aguirre
Irfan Ahmed
Michael Aiona
Amiethab Aiyer
Masao Akagi

Oslo, Norway
Fort Collins, CO
Philadelphia, PA
Los Angeles, CA
Rochester, MN
Lebanon, NH
St. Louis, MO
Zürich, Switzerland
Strasbourg, France
Ottawa, Canada
Iowa City, IA
Manchester, United Kingdom
Durham, NC
Pasadena, CA
Verona, Italy
St. Catharines, Canada
Bern, Switzerland
Bologna, Italy
San Antonio, TX
Mumbai, India
Zürich, Switzerland
Newark, NJ
Portland, OR
Miami, FL
Osaka, Japan

Yukio Akasaki
Behrooz Akbarnia
Keisuke Akiyama
Ozan Akkus
Venu Akuthota
Christoph Albers
Todd Albert
Frank Alberta
Eric Albrecht
John Albright
Julian Aldridge
Ilyas Aleem
Sheila Algan
Christian Allende
Daniel Allison
Louis Almekinders
David Altchek
Peter Amadio
Guillermo Ameer
Michael Amling
Nelly Andarawis-Puri
Andrew Anderson
Christopher Anderson
Colin Anderson
J. Robert Anderson

Fukuoka, Japan

La Jolla, CA

Kawachinagano, Japan

Cleveland, $\mathrm{OH}$

Denver, CO

Bern, Switzerland

New York, NY

Glen Rock, NJ

Lausanne, Switzerland

Iowa City, IA

Durham, NC

Ann Arbor, MI

Oklahoma City, OK

Cordoba, Argentina

Los Angeles, CA

Durham, NC

New York, NY

Rochester, MN

Evanston, IL

Hamburg, Germany

New York, NY

Salt Lake City, UT

Minneapolis, $M N$

Aurora, CO

Cleveland, $\mathrm{OH}$ 
Lucas Anderson

Megan Anderson

Paul Anderson

John Andrawis

Dimosthenis Andreou

Brett Andres

Jack Andrish

Irene Andrulis

Carolyn Anglin

Gregory Antonio

Samuel Antuna

Helen Anwander

Stephen Aoki

Luis Aponte-Tinao

Michael Archdeacon

Elizabeth Arendt

Jean-Noel Argenson

Javier Ariza

Douglas Armstrong

Hannu Aro

Asbjorn Aroen

James Aronson

Rohit Arora

Asaad Asaad

Apichat Asavamongkolkul

Janie Astephen-Wilson

Patrick Atkinson

Halil Atmaca

Takashi Atsumi

David Attarian

Brandon Ausk

Luke Austin

Matthew Austin

Alexander Avian

Hani Awad

Miguel Ayerza

George Babis

Geneva Baca

Christian Bach

John Bach

Kent Bachus

David Backstein

Sherry Backus

Marie Badalamente

Goo Hyun Baek

Marianne Baernholdt

Blaine Bafus

Chris Bailey

Champ Baker

Kevin Baker

Lisa Baker
Salt Lake City, UT

Boston, MA

Madison, WI

Torrance, $C A$

Münster, Germany

Offutt Air Force Base, NE

Cleveland, $\mathrm{OH}$

Toronto, Canada

Calgary, Canada

Hong Kong, China

Madrid, Spain

Bern, Switzerland

Salt Lake City, UT

Buenos Aires, Argentina

Cincinnati, $\mathrm{OH}$

Minneapolis, MN

Marseille, France

Barcelona, Spain

Hershey, PA

Turku, Finland

Lørenskog, Norway

Little Rock, AR

Innsbruck, Austria

London, United Kingdom

Bangkok, Thailand

Halifax, Canada

Flint, MI

Antalya, Turkey

Yokohama, Japan

Durham, NC

Seattle, WA

Egg Harbor Township, NJ

Philadelphia, PA

Graz, Austria

Rochester, NY

Buenos Aires, Argentina

Haidari, Greece

St. Louis, MO

Cologne, Germany

Newark, NJ

Salt Lake City, UT

Toronto, Canada

New York, NY

Stony Brook, NY

Seoul, Republic of Korea

Charlottesville, VA

Cleveland, $\mathrm{OH}$

Adelaide, Australia

Columbus, GA

Royal Oak, MI

Stamford, CT
Richard Baker

Andrea Baldini

Keith Baldwin

Massimiliano Baleani

Scott Ball

W. Timothy Ballard

Zsolt Balogh

Luke Balsamo

Philip Band

Ashok Banskota

Gilda Barabino

Thomas Barber

William Bargar

Brian Barlow

Douglas Barnes

Robert Barrack

Robert Barth

Alexandra Barthmann

Craig Bartlett

Yair Barzilay

Selda Başar

Kerem Basarir

Bryce Basques

Joshua Baumfeld

Keith Baumgarten

Judith Baumhauer

Joshua Baxter

Christopher Beauchamp

Paul Beaulé

Melanie Beaulieu

Joan Bechtold

Martin Beck

Asheesh Bedi

John Bednar

Burak Beksaç

Stephen Belkoff

Claudio Belvedere

Etienne Belzile

Elie Berbari

Pedro Beredjiklian

Aaron Berger

Richard Berger

Stephane Bergeron

Patrick Bergin

Marko Bergovec

Don Beringer

Joseph Bernstein

Nicky Bernthal

Boris Bershadsky

Nicholas Bertollo

David Beverland
Slaford, United Kingdom

Florence, Italy

Philadelphia, PA

Bologna, Italy

San Diego, CA

Chattanooga, TN

Newcastle, Australia

Portsmouth, VA

New York, NY

Kathmandu, Nepal

Atlanta, GA

Oakland, CA

Sacramento, CA

New York, NY

Houston, TX

St. Louis, MO

Chattanooga, TN

Ebensfeld, Germany

Burlington, VT

Jerusalem, Israel

Ankara, Turkey

Ankara, Turkey

Chicago, IL

Peabody, MA

Sioux Falls, SD

Rochester, NY

New York, NY

Scottsdale, AZ

Ottawa, Canada

Ann Arbor, MI

Minneapolis, MN

Luzern, Switzerland

Ann Arbor, MI

Cherry Hill, NJ

Istanbul, Turkey

Baltimore, MD

Bologna, Italy

Quebec City, Canada

Rochester, MD

Philadelphia, PA

Palo Alto, CA

Chicago, IL

Kirkland, Canada

Jackson, MS

Graz, Austria

Macon, GA

Philadelphia, PA

Santa Monica, CA

Minneapolis, $M N$

Dublin, Ireland

Belfast, United Kingdom 
Hari Bezwada

Mohit Bhandari

Timothy Bhattacharyya

Jacob Bickels

Sita Bierma-Zeinstra

Warren Bilker

Andrew Bing

Odion Binitie

Julius Bishop

Michael Bishop

Rudi Bitsch

Bernd Bittersohl

Sarah Bixby

John Blaha

R. Dale Blasier

Ralph Blasier

Karen Bloch

Roy Bloebaum

Taco Blokhuis

Jesper Blomquist

Thomas Blumenfeld

Beata Bode-Lesniewska

Paul Boehm

Friedrich Boettner

Kath Bogie

Eric Bogner

Aaron Bois

Patrick Boland

Robert Bolash

Santiago Bongiovanni

Christopher Bono

Peter Bonutti

Robert Booth, Jr.

Barbara Bordini

Jorge Boretto

Joseph Borrelli

Arjan Bot

Elisabeth Botelho-Nevers

Michael Bourne

Brett Boutin

William Bowman

Matison Boyer

Kevin Bozic

Jennifer Brach

Laurence Bradley

Jonathan Braman

Maria Luisa Brandi

Eric Brandser

Miriam Bredella

Gary Brenner

Steffen Breusch
Philadelphia, PA

Hamilton, Canada

Bethesda, MD

Tel-Aviv, Israel

Rotterdam, Netherlands

Philadelphia, PA

Oswestry, United Kingdom

Tampa, FL

Redwood City, CA

Memphis, TN

Heidelberg, Germany

Düsseldorf, Germany

Boston, MA

Ann Arbor, MI

Little Rock, AR

Escanaba, MI

Nashville, TN

Salt Lake City, UT

Utrecht, Netherlands

Bergen, Norway

Sacramento, CA

Zürich, Switzerland

Nuremberg, Germany

New York, $N Y$

Cleveland, $\mathrm{OH}$

New York, NY

Calgary, Canada

New York, NY

Cleveland, $\mathrm{OH}$

Buenos Aires, Argentina

Boston, MA

Effingham, IL

Philadelphia, PA

Bologna, Italy

Buenos Aires, Argentina

Arlington, TX

Amsterdam, Netherlands

St. Etienne, France

Salt Lake City, UT

Phoenix, AZ

San Diego, $C A$

Charleston, SC

Austin, TX

Pittsburgh, PA

Birmingham, AL

Minneapolis, $M N$

Florence, Italy

Cincinnati, $\mathrm{OH}$

Boston, MA

Boston, MA

Edinburgh, United Kingdom
Brian Brigman

Silviu Brill

Mats Brittberg

James Brodsky

Henry Broekhuyse

Robert Brophy

Stig Brorson

Reinoud Brouwer

Haydee Brown

Kenneth Brown

T. Desmond Brown

William Brox

Wendy Bruinsma

Reinald Brunner

Laura Bruse

Lorenz Büchler

William Bugbee

Geert Buijze

Leonard Buller

Wilton Bunch

Bruce Bunnell

Alissa Burge

Rolf Burghardt

J. Kenneth Burkus

Albert Burstein

Michael Buschmann

Daniel Buss

Jason Busse

Erin Butler

Martin Buttaro

J. W. Thomas Byrd

Miguel Cabanela

Guy Cafri

Vincent Caiozzo

Michelle Caird

Joe Calandra

Maurizio Calcagni

Ryan Calfee

Tilman Calliess

George Calvert

Domenico Campanacci

David Campbell

Mitchell Campbell

Patricia Campbell

T. Mark Campbell

Lisa Cannada

Rodolfo Capanna

William Capello

Gianluca Cappelleri

Julio Carballido-Gamio

James Carey
Durham, NC

Tel-Hashomer, Israel

Kungsbacka, Sweden

Dallas, TX

Vancouver, Canada

Chesterfield, MO

Herlev, Denmark

Groningen, Netherlands

New York, NY

Vancouver, Canada

Boston, MA

Fresno, $C A$

Amsterdam, Netherlands

Basel, Switzerland

Henderson, $N \mathrm{~V}$

Bern, Switzerland

La Jolla, CA

Amsterdam, Netherlands

Miami, FL

Birmingham, AL

New Orleans, LA

New York, $N Y$

Hamburg, Germany

Columbus, $\mathrm{OH}$

Sarasota, FL

Montreal, Canada

Edina, $M N$

Hamilton, Canada

Boston, MA

Buenos Aires, Argentina

Nashville, TN

Rochester, MN

San Diego, CA

Irvine, $C A$

Ann Arbor, MI

Mount Pleasant, SC

Zürich, Switzerland

St. Louis, MO

Hannover, Germany

Duarte, $C A$

Firenze, Italy

Adelaide, Australia

Louisville, $K Y$

Los Angeles, CA

Ottawa, Canada

St. Louis, MO

Firenze, Italy

Indianapolis, IN

Milano, Italy

San Francisco, CA

Philadelphia, PA 


\begin{tabular}{|c|c|c|c|}
\hline Alberto Carli & New York, $N Y$ & Alice Chu & New York, $N Y$ \\
\hline Cathy Carlson & St. Paul, $M N$ & Yong-Min Chun & Seoul, Republic of Korea \\
\hline Walter Carlson & Sioux Falls, SD & William Ciccone, II & Colorado Springs, $\mathrm{CO}$ \\
\hline Åke Carlsson & Lund, Sweden & Cara Cipriano & St. Louis, MO \\
\hline Michael Carmont & Telford, United Kingdom & Henry Clarke & Scottsdale, AZ \\
\hline Bradley Carofino & Virginia Beach, VA & John Clarke & Philadelphia, $P A$ \\
\hline Leah Carreon & Louisville, $K Y$ & Michael Clarke & Syracuse, $N Y$ \\
\hline Robert Carrigan & Philadelphia, $P A$ & Martin Clauss & Liestal, Switzerland \\
\hline Charles Cassidy & Boston, MA & Robert Closkey & Tom's River, NJ \\
\hline Pablo Castañeda & Lomas De Chapultepec, Mexico & Terry Clyburn & Houston, $T X$ \\
\hline Vincenzo Castellano & New York, $N Y$ & Melanie Coathup & Stanmore, United Kingdom \\
\hline Frank Castro & Louisville, $K Y$ & R. Gregory Cochran & San Francisco, $C A$ \\
\hline Fabio Catani & Rome, Italy & Steven Cohen & Philadelphia, PA \\
\hline Francois Cazeneuve & Laon, France & Brian Cole & Chicago, $I L$ \\
\hline Derya Çelik & Istanbul, Turkey & Judith Cole & Memphis, TN \\
\hline Simone Cenci & Milano, Italy & John Collier & Hanover, $\mathrm{NH}$ \\
\hline Jaskarndip Chahal & Toronto, Canada & Matthew Collier & Park City, UT \\
\hline Aaron Chamberlain & St. Louis, MO & Dennis Collis & Eugene, $O R$ \\
\hline Coonoor Chandrasekar & Liverpool, United Kingdom & Matthew Colman & Boston, MA \\
\hline Srinivasan Chandrasekhar & Indianapolis, IN & Clifford Colwell, Jr. & La Jolla, CA \\
\hline Chong Bum Chang & Seoul, Republic of Korea & Eithne Comerford & Neston, United Kingdom \\
\hline Lin Chang & Los Angeles, $C A$ & Thomas Comfort & Stillwater, $M N$ \\
\hline Roland Chang & Chicago, IL & Susan Connolly & Boston, MA \\
\hline Howard Chansky & Seattle, WA & Stan Conte & Los Angeles, $C A$ \\
\hline Debra Charlesworth & Houghton, MI & James Cook & Columbia, $M O$ \\
\hline Ajit Chaudhari & Columbus, $\mathrm{OH}$ & Theodore Cooke & Kingston, Canada \\
\hline Sonia Chaudhry & Hartford, $C T$ & H. John Cooper & New York, $N Y$ \\
\hline Caroline Chebli & Sarasota, $F L$ & Reginald Cooper & Iowa City, IA \\
\hline Jacques Chelly & Pittsburgh, PA & Lawson Copley & Dallas, $T X$ \\
\hline Antonia Chen & Philadelphia, PA & Frank Cordasco & New York, $N Y$ \\
\hline Neal Chen & Ann Arbor, MI & José Cordero-Ampuero & Tres Cantos, Spain \\
\hline Qi-xin Chen & Hangzhou, China & Charles Cornell & New York, $N Y$ \\
\hline Shiyi Chen & Shanghai, China & Christopher Cornett & Omaha, NE \\
\hline Tien-Hua Chen & Taipei, Taiwan & Roger Cornwall & Cincinnati, $\mathrm{OH}$ \\
\hline Wei Ming Chen & Taipei, Taiwan & Costantino Corradini & Milano, Italy \\
\hline Cheng-Kung Cheng & Taipei, Taiwan & Stephane Corvec & Nantes, France \\
\hline David Cheong & Tampa, FL & Michael Coscia & Indianapolis, IN \\
\hline Stéphane Cherix & Lausanne, Switzerland & Aidan Cosgrove & Belfast, United Kingdom \\
\hline Tim Chesser & Bristol, United Kingdom & Matias Costa-Paz & Buenos Aires, Argentina \\
\hline Felix Cheung & Huntington, $W V$ & John Costerton & Los Angeles, $C A$ \\
\hline Jason Pui Yin Cheung & Hong Kong, China & Patrick Costigan & Kingston, Canada \\
\hline Kin-wing Cheung & Hong Kong, China & Hayden Courtland & New York, $N Y$ \\
\hline Wing-Hoi Cheung & Hong Kong, China & Paul Courtney & Philadelphia, PA \\
\hline François Chevalley & Lausanne, Switzerland & Aurélien Courvoisier & Grenoble, France \\
\hline Avneesh Chhabra & Dallas, $T X$ & Andrea Cracchiolo, III & Santa Monica, CA \\
\hline Ki Chin & Toronto, Canada & Peter Cram & Toronto, Canada \\
\hline Kingsley Chin & Fort Lauderdale, FL & Xavier Crevoisier & Lausanne, Switzerland \\
\hline Alexander Ching & Portland, $O R$ & Joseph Crisco, III & Providence, $R I$ \\
\hline Mary Chisholm & New York, $N Y$ & Brett Crist & Columbia, $M O$ \\
\hline Samuel Cho & New York, $N Y$ & William Cross, III & Rochester, $M N$ \\
\hline Yoon Je Cho & Seoul, Republic of Korea & John Cuckler & Naples, FL \\
\hline
\end{tabular}


Quanjun Cui

Nathan Cummins

Barbara Currier

Mark DaCambra

Zoe Dailiana

Jean-Claude D'Alleyrand

James D'Antonio

Manuel DaSilva

Jean-Pierre David

Darin Davidson

Jeffrey Davila

Kirkland Davis

Thomas Davis

Tim Davis

Charles Davis, III

Judd Day

Joseph De Beer

Joost de Bruijn

Ivan De Martino

Lieven De Wilde

Richard Debski

Thomas DeCoster

Vincent Deeney

Kamal Deep

Ilse Degreef

Henry DeGroot

Carl Deirmengian

Gregory Deirmengian

Ronald Delanois

Christian Delaunay

Alberto Delgado-Martinez

Gregory Della Rocca

Craig Della Valle

Matthew Delmonico

Demetris Delos

Marlene DeMaio

Xiang-Hua Deng

Douglas Dennis

Geoffrey Dervin

Kathleen Derwin

Peter Devane

Vedat Deviren

Anand Devnani

Alberto Di Martino

Stephanie Di Stasi Roewer

Veronica Diaz

Paul DiCesare

Kyle Dickson

Matthew Dietz

Albrecht Dietze

Gregory DiFelice
Charlottesville, VA

Rochester, MN

Hanover, $\mathrm{NH}$

New Westminster, Canada

Larissa, Greece

Bethesda, MD

Moon Township, PA

Providence, RI

Erlangen, Germany

Seattle, WA

Bethesda, MD

Madison, WI

Silver Spring, $M D$

Nottingham, United Kingdom

Hershey, PA

Philadelphia, PA

Cape Town, South Africa

London, United Kingdom

New York, NY

Gent, Belgium

Pittsburgh, PA

Albuquerque, NM

Pittsburgh, PA

Glasgow, United Kingdom

Pellenberg, Belgium

Newton, MA

Wynnewood, PA

Philadelphia, PA

Baltimore, $M D$

Longjumeau, France

Jaen, Spain

Columbia, MO

Chicago, IL

Kingston, RI

New York, $N Y$

Philadelphia, PA

New York, $N Y$

Denver, $C O$

Ottawa, Canada

Cleveland, $\mathrm{OH}$

Wellington South, New Zealand

San Francisco, $C A$

Serdang, Malaysia

Rome, Italy

Columbus, $\mathrm{OH}$

Miami, FL

Sacramento, CA

Houston, TX

Morgantown, $W V$

Rostock, Germany

New York, $N Y$
Abhijit Dighe

Sander Dijkstra

Joshua Dines

Changhai Ding

Ming Ding

Darryl D'Lima

Seth Dodds

Mathieu Domalain

Davide Donati

Claudio Dora

Frederick Dorey

Stephen Doty

Paul Dougherty

Klaus Draenert

Lorenzo Drago

Thomas Dreher

Jacob Drew

M. Hicham Drissi

Adam Dubrowski

Shivi Duggal

Sukhdeep Dulai

Gregory Dumanian

Charles Dumont

Michael Dunn

Robert Dunsmuir

Xavier Duralde

Devendra Dusane

Anil Dutta

Paul Duwelius

Maureen Dwyer

Scott Dye

George Dyer

Brandon Earp

Deborah Eastwood

Nabil Ebraheim

Edward Ebramzadeh

Malcolm Ecker

Timo Ecker

Donald Eckhoff

Walter Eckman

J. Gordon Edelson

Av Edidin

T. Bradley Edwards

Matthieu Ehlinger

Garth Ehrlich

Neal ElAttrache

Ron El-Hawary

Pernilla Eliasson

James Elliott

Amr ElMaraghy

Cynthia Emory
Charlottesville, VA

Leiden, Netherlands

New York, NY

Tasmania, Australia

Odense, Denmark

La Jolla, CA

Miami, FL

Cedex, France

Bologna, Italy

Zürich, Switzerland

Los Angeles, CA

New York, NY

Jacksonville, FL

Munich, Germany

Milan, Italy

Heidelberg, Germany

Boston, MA

Farmington, CT

Toronto, Canada

Hartsdale, NY

Edmonton, Canada

Chicago, IL

Zürich, Switzerland

New Brunswick, NJ

Leeds, United Kingdom

Atlanta, GA

Columbus, $\mathrm{OH}$

San Antonio, TX

Portland, OR

Newton, MA

San Francisco, $C A$

Boston, MA

Boston, MA

London, United Kingdom

Toledo, $\mathrm{OH}$

Los Angeles, CA

Philadelphia, PA

Bern, Switzerland

Aurora, CO

Tupelo, MS

Tiberias, Israel

Redwood City, CA

Houston, TX

Strasbourg, France

Philadelphia, PA

Los Angeles, CA

Halifax, Canada

Copenhagen, Denmark

Cambridge, United Kingdom

Toronto, Canada

Winston Salem, NC 
Yoshimi Endo

C. Anderson Engh, Jr.

F. Kayser Enneking

Motomi Enomoto-Iwamoto

Steven Eppell

Howard Epps

Cevat Erisken

Saliha Eroglu Demir

Thomas Errico

Antti Eskelinen

Nuria Espallargas

Christina Esposito

Christopher Evans

Richard Evans

Andrea Evenski

Denise Eygendaal

Nicola Fabbri

Cesare Faldini

Florian Fankhauser

Sara Farquhar

Jack Farr

Sebastian Farr

David Farrington

Luc Favard

Angela Fearon

Keith Fehring

Thomas Fehring

Edward Fehringer

Joseph Feinberg

Frieda Feldman

David Felson

Peter Fennema

Peter Ferguson

Donald Ferlic

Stefano Ferrari

Kara Fields

Mark Figgie

Stephanie Filbay

Blair Filler

Aharon Finestone

Bernd Fink

David Fisher

Steven Fitzgerald

Braden Fleming

Nicholas Fletcher

Gunnar Flivik

Jeremy Fogelson

Pedro Foguet

Franco Folli

Clary Foote

Matteo Formica
New York, NY

Alexandria, $V A$

Gainesville, $F L$

Philadelphia, PA

Cleveland, $\mathrm{OH}$

Houston, TX

New York, NY

Istanbul, Turkey

New York, NY

Tampere, Finland

Birkeland, Norway

New York, NY

Boston, MA

Erie, $\mathrm{CO}$

Philadelphia, PA

Breda, Netherlands

New York, NY

Bologna, Italy

Graz, Austria

Alexandria, VA

Indianapolis, IN

Vienna, Austria

Bormujos, Spain

Tours, France

Garran, Australia

Richmond, VA

Charlotte, NC

Omaha, NE

New York, NY

New York, NY

Boston, MA

Dietikon, Switzerland

Toronto, Canada

Denver, $C O$

Bologna, Italy

New York, NY

New York, $N Y$

Oxford, United Kingdom

Los Angeles, CA

Reut, Israel

Markgroeningen, Germany

Indianapolis, IN

Cleveland, $\mathrm{OH}$

Providence, RI

Atlanta, GA

Lund, Sweden

Rochester, MN

Coventry, United Kingdom

San Antonio, TX

Hamilton, Canada

Genova, Italy
Christopher Forthman

John Fowler

Austin Fragomen

Matthias Frank

Mark Frankle

Orrin Franko

Michael Freehill

Thomas Freude

Mark Friedberg

Eitan Friedman

Richard Friedman

Max Friedrich

Michael Frink

J. Christopher Fritton

John Froelich

Todd Frush

Bruno Fuchs

Renee Fuhrmann

Masanori Fujii

Tadashi Fujii

Takuya Fujimoto

Masatoshi Fujiwara

Kanji Fukuda

Philipp Funovics

Ove Furnes

Keith Gabriel

Olivier Gagey

Michael Gagnon

Barry Gainor

Kyle Galles

Jiri Gallo

Kazuyoshi Gamada

Theodore Ganley

Reinhold Ganz

Donald Garbuz

Africa García-Orad

Eduardo García-Rey

Michael Gardner

Göran Garellick

Steven Garfin

Nitin Garg

Jonathan Garino

Douglas Garland

Grant Garrigues

Nathaly Gaudreault

Ralph Gaulke

Frances Gavelli

Gregory Gebauer

Sebastian Gehmert

Thorsten Gehrke

Jonathan Gelber
Lutherville, $M D$

Pittsburgh, PA

New York, NY

Greifswald, Germany

Temple Terrace, FL

San Diego, CA

Winston Salem, NC

Tuebingen, Germany

Santa Monica, CA

Tel-Hashomer, Israel

Charleston, SC

Bonn, Germany

Marburg, Germany

Newark, NJ

Aurora, CO

Southfield, MI

Zürich, Switzerland

Bad Neustadt, Germany

Yahatanishi-ku, Japan

Kashiba, Japan

Akashi, Japan

Kobe City, Japan

Osaka, Japan

Vienna, Austria

Bergen, Norway

Springfield, IL

le Kremlin-Bicetre, France

Durham, NC

Columbia, $\mathrm{MO}$

Des Moines, IA

Olomouc, Czech Republic

Higashi-Hiroshima, Japan

Philadelphia, PA

Guemligen, Switzerland

Vancouver, Canada

Leioa, Spain

Madrid, Spain

Palo Alto, CA

Göteborg, Sweden

San Diego, CA

Bhopal, India

Malvern, PA

Long Beach, CA

Durham, NC

Quebec, Canada

Hannover, Germany

Bethesda, MD

Philadelphia, PA

Basel, Switzerland

Hamburg, Germany

New York, NY 


\begin{tabular}{|c|c|c|c|}
\hline Jeffrey Geller & New York, $N Y$ & Samuel Gourion-Arsiquaud & Princeton, $N J$ \\
\hline Steven George & Gainesville, $F L$ & Gregory Grabowski & Columbia, SC \\
\hline Andrew Georgiadis & St. Paul, MN & Daniel Grande & Manhasset, $N Y$ \\
\hline Paul Gerdhem & Stockholm, Sweden & Froilan Granero-Molto & Pamplona, Spain \\
\hline Craig Gerrand & Newcastle upon Tyne, United Kingdom & Chancellor Gray & Gainesville, FL \\
\hline David Gershuni & Zichron Ya'akov, Israel & Andrew Green & Providence, $R I$ \\
\hline Patrick Getty & Cleveland, $\mathrm{OH}$ & John Green & Seattle, WA \\
\hline Charles Getz & Philadelphia, PA & Stuart Green & Orange, $C A$ \\
\hline Jeroen Geurts & Basel, Switzerland & David Greenberg & St. Louis, MO \\
\hline Michelle Ghert & Hamilton, Canada & Todd Greenberg & Seattle, WA \\
\hline Hassan Ghomrawi & Chicago, IL & Edward Greenfield & Cleveland, $\mathrm{OH}$ \\
\hline Cecilia Giachelli & Seattle, WA & A. Seth Greenwald & Cleveland, $\mathrm{OH}$ \\
\hline Philip Giampietro & Madison, WI & Justin Greisberg & New York, $N Y$ \\
\hline Charles Giangarra & Huntington, $W V$ & Ronald Grelsamer & New York, $N Y$ \\
\hline Sandro Giannini & Bologna, Italy & Phillip Gribble & Toledo, $\mathrm{OH}$ \\
\hline C. Parker Gibbs & Gainesville, $F L$ & Frank Griffin & Van Buren, AR \\
\hline Gary Gibson & Detroit, $M I$ & Letha Griffin & Atlanta, $G A$ \\
\hline Monique Gignac & Toronto, Canada & William Griffin & Charlotte, NC \\
\hline Jeremy Gilbert & Syracuse, $N Y$ & Robert Grimer & Birmingham, United Kingdom \\
\hline Joshua Giles & London, United Kingdom & Kirill Gromov & Hvidovre, Denmark \\
\hline Harinderjit Gill & Oxford, United Kingdom & Allan Gross & Toronto, Canada \\
\hline J. Brian Gill & Omaha, NE & R. Michael Gross & Omaha, $N E$ \\
\hline Thomas Gill & Boston, MA & Ted Gross & Seattle, WA \\
\hline Robert Gillespie & Cleveland, $\mathrm{OH}$ & Lars Grossterlinden & Hamburg, Germany \\
\hline Jeffrey Gimble & Baton Rouge, LA & Claudia Grothe & Hannover, Germany \\
\hline Nicholas Giori & Palo Alto, $C A$ & Helen Gruber & Charlotte, $N C$ \\
\hline Julien Girard & Lille, France & Alexander Grübl & Vienna, Austria \\
\hline Federico Girardi & New York, $N Y$ & Thomas Grupp & Tuttlingen, Germany \\
\hline Panagiotis Givissis & Thessaloniki, Greece & Scott Guelcher & Nashville, TN \\
\hline Michael Glanzmann & Zürich, Switzerland & Zbigniew Gugala & Galveston, $T X$ \\
\hline John Glaser & Charleston, SC & Lawrence Gulotta & New York, $N Y$ \\
\hline Jonathan Glashow & New York, $N Y$ & Klaus-Peter Günther & Dresden, Germany \\
\hline Andrew Glassman & Columbus, $\mathrm{OH}$ & Sergio Gutierrez & Tampa, FL \\
\hline Richard Glisson & Durham, $N C$ & Gregory Guyton & Baltimore, $M D$ \\
\hline Vijay Goel & Toledo, $\mathrm{OH}$ & Clemens Gwinner & Berlin, Germany \\
\hline Wade Gofton & Ottawa, Canada & Steven Haas & New York, $N Y$ \\
\hline Robert Goitz & Pittsburgh, PA & Carl Haasper & Hamburg, Germany \\
\hline Charles Goldfarb & St. Louis, MO & Donald Hackbarth & Milwaukee, WI \\
\hline Stanley Goldsmith & New York, $N Y$ & Michael Hadjiargyrou & Stony Brook, NY \\
\hline Christina Goldstein & Toronto, Canada & Mahmoud Hafez & Cairo, Egypt \\
\hline Enrique Gomez-Barrena & Madrid, Spain & Frederic Hafferty & Duluth, $M N$ \\
\hline Hyun Sik Gong & Seongnam-si, Republic of Korea & Jennifer Hagen & Gainesville, $F L$ \\
\hline Yao-Qin Gong & Jinan, China & Elisabet Hagert & Stockholm, Sweden \\
\hline Stuart Goodman & Redwood City, CA & Warren Haggard & Memphis, TN \\
\hline J. Eric Gordon & St. Louis, $M O$ & Andrew Haims & New Haven, $C T$ \\
\hline Max Gordon & Stockholm, Sweden & David Hak & Denver, $\mathrm{CO}$ \\
\hline Zachary Gordon & Cleveland, $\mathrm{OH}$ & Matthew Halanski & Madison, WI \\
\hline Masafumi Gotoh & Kurume, Japan & Deborah Hall & Chicago, IL \\
\hline Frank Gottschalk & Dallas, $T X$ & Mederic Hall & Iowa City, IA \\
\hline Nikolaos Gougoulias & Camberley, United Kingdom & Brian Hallstrom & Ann Arbor, $M I$ \\
\hline Francois Gouin & Nantes, France & Reggie Hamdy & Montreal, Canada \\
\hline
\end{tabular}


David Hamilton

James Hamilton

Sommer Hammoud

Takehito Hananouchi

Douglas Hanel

Markus Hanke

Edward Hanley

Arlen Hanssen

Knut Harboe

Jendrik Hardes

Brian Harfe

Hirotaka Haro

Ian Harris

Janette Harro

David Hart

Craig Hartrick

Andrew Haskell

Miroslav Haspl

Leslie Hausmann

Richard Hawkins

John Healey

William Healy

Robert Heck

R. David Heekin

Petra Heesterbeek

Michael Heggeness

John Heiner

Adam Heish

Marco Helder

Markus Heller

Brad Hendershot

Eric Henderson

James Henderson

Marilyn Heng

Heath Henninger

William Hennrikus

Joseph Hentz

Matthew Hepinstall

Volkmar Heppert

Thomas Hering

James Herndon

J. Anthony Herring

Sebastian Herrmann

Dolfi Herscovici, Jr.

Iftach Hetsroni

Timothy Hewett

Thomas Heyse

Benton Heyworth

Noreen Hickok

Kevin Hildebrand

Caroline Hing
Edinburgh, United Kingdom

Portland, OR

New York, NY

Daito, Japan

Seattle, WA

Bern, Switzerland

Charlotte, NC

Rochester, $M N$

Stavanger, Norway

Münster, Germany

Gainesville, FL

Chuo, Japan

Liverpool, Australia

Baltimore, $M D$

Calgary, Canada

Troy, MI

San Carlos, CA

Krapinske Toplice, Croatia

Pittsburgh, PA

Greenville, $S C$

New York, NY

Burlington, $M A$

Memphis, TN

Jacksonville, FL

Nijmegen, Netherlands

Houston, TX

Madison, WI

College Park, MD

Amsterdam, Netherlands

Berlin, Germany

Bethesda, MD

Hanover, $\mathrm{NH}$

Syracuse, $N Y$

Boston, MA

Salt Lake City, UT

Hershey, PA

Scottsdale, AZ

New York, $N Y$

Ludwigshafen, Denmark

Cleveland, $\mathrm{OH}$

Boston, MA

Dallas, TX

Berlin, Germany

Temple Terrace, FL

Kfar Saba, Israel

Rochester, MN

Marburg, Germany

Boston, MA

Philadelphia, PA

Calgary, Canada

London, United Kingdom
Andreas Hingsammer

Beat Hintermann

Bruce Hirsch

Henry Ho

W. Andrew Hodge

Richard Hodgson

Pierre Hoffmeyer

Siegfried Hofmann

MaCalus Hogan

Thomas Hogervorst

Nilsson Holguin

Paul Holman

Lukas Holzer

Gary Hooper

John Ty Hopkins

Sevan Hopyan

Daniel Horton

Harish Hosalkar

Gamal Hosny

Noboru Hosono

Peter Houghton

Stephen Howell

Andrew Howlett

Alan Hreljac

Chun-Hsiung Huang

Leaf Huang

Tsung-Jen Huang

James Huddleston

Paul Huddleston

Richard Hughes

Maury Hull

Eric Hume

Clark Hung

Man Hung

Michael Huo

Christof Hurschler

Shepard Hurwitz

Ali Hussam

Wei-Ting Hwang

John Hyndman

Joseph Iannotti

Makoto Ieguchi

Thomas Ilchmann

Shinji Imade

Carl Imhauser

Yutaka Inaba

Maria Inacio

Stephen Incavo

Eileen Ingham

Bernardo Innocenti

Massimo Innocenti
Boston, MA

Basel, Switzerland

Philadelphia, PA

Alexandria, VA

West Palm Beach, FL

Manchester, United Kingdom

Geneva, Switzerland

Stolzalpe, Austria

Pittsburgh, PA

Den Haag, Netherlands

St. Louis, MO

Houston, TX

Graz, Austria

Christchurch, New Zealand

Provo, UT

Toronto, Canada

New Brunswick, NJ

National City, CA

Cairo, Egypt

Osaka, Japan

San Antonio, TX

Sacramento, $C A$

Spokane, WA

Sacramento, CA

Taipei, Taiwan

Chapel Hill, NC

Chiayi, Taiwan

Stanford, CA

Rochester, MN

Ann Arbor, MI

Davis, CA

Philadelphia, PA

New York, NY

Salt Lake City, UT

Dallas, TX

Hannover, Germany

Chapel Hill, NC

Columbia, $M O$

Philadelphia, PA

Halifax, Canada

Cleveland, $\mathrm{OH}$

Izumi, Japan

Münchenstein, Switzerland

Izumo, Japan

New York, $N Y$

Yokohama, Japan

Adelaide, Australia

Houston, TX

Leeds, United Kingdom

Bruxelles, Belgium

Firenze, Italy 
Nozomu Inoue

Richard Iorio

Ernesto Ippolito

Farhad Iranpour

Jonathan Isaacs

Michael Isakoff

Yasuyuki Ishibashi

Hiromu Ito

Yoichi Ito

Eijii Itoi

Yukihide Iwamoto

Norimasa Iwasaki

Wesley Jackson

Cale Jacobs

Michael Jacobs

Fredrick Jaffe

Kenneth Jaffe

Thomas Linding Jakobsen

Bilal Jamal

Amir Jamali

Esa Jämsen

Tjin-Shing Jap

Branislav Jaramaz

Jacob Jaremko

Abbas Jawad

Shari Jawetz

Prakash Jayabalan

Peter Jebson

W. Scott Jellish

Jessica Jennings

Louise Jennings

Jean-Yves Jenny

Dae-Geun Jeon

Kyle Jeray

Brian Jewett

Sujee Jeyapalina

Ramon Jimenez

Li Jin

Anthony Johnson

David Johnson

Mark Johnson

Randon Johnson

Brian Johnstone

Eric Jones

Kevin Jones

Kristofer Jones

Lynne Jones

Michael Jones

Morgan Jones

Susan Joy

Thomas Joyce
Chicago, IL

New York, NY

Rome, Italy

London, United Kingdom

Richmond, VA

Hartford, CT

Aomori, Japan

Kyoto, Japan

Osaka, Japan

Sendai, Japan

Fukuoka, Japan

Sapporo, Japan

Bethesda, MD

Lexington, $K Y$

Baltimore, $M D$

New York, $N Y$

Birmingham, AL

Hvidovre, Denmark

Glasgow, United Kingdom

Sacramento, $C A$

Tampere, Finland

Taipei, Taiwan

Pittsburgh, PA

Edmonton, Canada

Philadelphia, PA

New York, $N Y$

Columbia, $M O$

Grand Rapids, MI

Maywood, IL

Memphis, TN

Leeds, United Kingdom

Illkirch, France

Seoul, Republic of Korea

Greenville, SC

Eugene, OR

Salt Lake City, UT

Monterey, CA

Charlottesville, VA

Fort Sam Houston, San Antonio, TX

Bristol, United Kingdom

Kansas City, MO

Omaha, NE

Portland, OR

Detroit, $M I$

Salt Lake City, UT

New York, NY

Baltimore, $M D$

Chicago, IL

Cleveland, $\mathrm{OH}$

Independence, $\mathrm{OH}$

Newcastle upon Tyne, United Kingdom
Kethy Jules-Elysee

Jesse Jupiter

Cristina Justice

Scott Kaar

Abbott Kagan

Leslie Kalish

Robin Kamal

Makoto Kamegaya

Leonid Kandel

Robert Kane

Lana Kang

Chia-Hung Kao

Eleni Kapreli

Mustafa Karahan

Matthew Karam

Johan Kärrholm

Richard Kasch

Masafumi Kashii

Stephen Kates

John Kaufman

Kenton Kaufman

Yoshiharu Kawaguchi

Mamoru Kawakami

Hirotaka Kawano

Robert Kay

Stephen Kearns

Tony Keaveny

James Keene

Jay Keener

James Keeney

Henrik Kehlet

James Kellam

Scott Kelley

Cynthia Kelly

James Kelly

John Kelly IV

Benjamin Kendrick

John Kennedy

Stephen Kennedy

Hayrettin Kesmezacar

Oliver Kessler

Monti Khatod

Joseph Khoury

Ata Kiapour

Piya Kiatisevi

Louis Kidder

Han Jo Kim

Han-Soo Kim

Harry Kim

Jae Kwang Kim

Sae Hoon Kim
New York, NY

Boston, MA

Bethesda, MD

St. Louis, $M O$

Fort Myers, FL

Boston, MA

Redwood City, CA

Chiba, Japan

Jerusalem, Israel

Minneapolis, MN

New York, NY

Taichung, Taiwan

Athens, Greece

Istanbul, Turkey

Iowa City, IA

Goteborg, Sweden

Greifswald, Germany

Suita, Japan

Richmond, VA

Santa Clarita, CA

Rochester, MN

Toyama, Japan

Wakayama, Japan

Tokyo, Japan

Los Angeles, CA

Galway, Ireland

Berkeley, CA

Madison, WI

St. Louis, $M O$

Columbia, $\mathrm{MO}$

Copenhagen, Denmark

Houston, TX

Durham, NC

Denver, $C O$

San Francisco, $C A$

Philadelphia, PA

Oxford, United Kingdom

New York, NY

Seattle, WA

Istanbul, Turkey

Zürich, Switzerland

Baldwin Park, CA

Birmingham, $A L$

Boston, MA

Bangkok, Thailand

Minneapolis, MN

New York, NY

Seoul, Republic of Korea

Dallas, TX

Seoul, Republic of Korea

Seoul, Republic of Korea 
Shin-Yoon Kim

Tae Kyun Kim

Won Joong Kim

Yong Sik Kim

Young-Kyu Kim

John Kincaid

Karen King

Stefan Kinkel

J. Kinnett

Humberto Kitadai

Aarne Kivioja

Christian Kleber

Gregg Klein

Michael Klein

Eric Klineberg

Stephen Klinge

Hans-Michael Klinger

Peter Kloen

Jih-Yang Ko

Naomi Kobayashi

Seneki Kobayashi

Werner Koehnlein

Hideyuki Koga

Andres Kohan

Yusuke Kohno

E. Anders Kolb

Søren Kold

David Komatsu

Richard Komistek

Gabrielle Konin

Feza Korkusuz

Anastasios Korompilias

Panagiotis Korovessis

Tomihasa Koshino

Panagiotis Koulouvaris

Melissa Kounine

Veronika Kralj-Iglič

Walter Krengel

Petra Krepler

Andreas Krieg

James Krieg

Leif Pål Kristiansen

Aaron Krych

Todd Kuiken

Julia Kulkova

Hiroshi Kuroki

Steven Kurtz

Bong Cheol Kwon

Brian Kwon

Sae-Kwang Kwon

Gerold Labek
Daegu, Republic of Korea

Seongnam-Si, Republic of Korea

Seoul, Republic of Korea

Seoul, Republic of Korea

Incheon, Republic of Korea

Indianapolis, IN

Aurora, $\mathrm{CO}$

Heidelberg, Germany

New Orleans, LA

São Paulo, Brazil

Helsinki, Finland

Berlin, Germany

Paramus, NJ

New York, $N Y$

Sacramento, CA

Providence, RI

Goettingen, Germany

Amsterdam, Netherlands

Kaohsiung Hsien, Taiwan

Yokohama, Japan

Nagano, Japan

Geneva, Switzerland

Tokyo, Japan

Caba, Argentina

Fukuoka, Japan

Wilmington, DE

Aarhus, Denmark

Stony Brook, NY

Knoxville, TN

New York, NY

Ankara, Turkey

Ioannina, Greece

Patras, Greece

Yokohama, Japan

Athens, Greece

Toledo, $\mathrm{OH}$

Ljubljana, Slovenia

Seattle, WA

Vienna, Austria

Basel, Switzerland

Seattle, WA

Oslo, Norway

Rochester, MN

Chicago, IL

Turku, Finland

Miyazaki, Japan

Philadelphia, PA

Anyang, Republic of Korea

Boston, MA

Bucheon si, Republic of Korea

Innsbruck, Austria
Amy Ladd

Amitabha Lahiri

Claudette Lajam

Spencer Lake

Dror Lakstein

Jerome Lambert

Kenneth Lamp

Bruce Landon

Glenn Landon

Franz Landsiedl

Joseph Lane

Odette Laneuville

Brent Lanting

Jean-Denis Laredo

A. Noelle Larson

Paul LaStayo

L. Daniel Latt

Cato Laurencin

Stephane Lavallee

Patricia Lavand'homme

Carlos Lavernia

Christopher Lavy

Keith Lawhorn

Ericka Lawler

McKinley Lawson

Jeffrey Lawton

Peter Laz

Stergios Lazarinis

Alberto Leardini

Lee Leddy

Charles Ledonio

Brendan Lee

Donald Lee

Francis Lee

Kyoung Min Lee

Sang-Hun Lee

Jeff Leiter

Lawrence Lenke

Linda LeResche

Daniel Lerman

Tesfaye Leta

Michael Leunig

Marc Levenston

Adam Levin

Paul Levin

Ari Levine

Brett Levine

Bruce Levy

Jonathan Levy

Roger Levy

Gladius Lewis
Palo Alto, CA

Singapore, Singapore

New York, NY

St. Louis, MO

Petah-Tikva, Israel

Paris, France

Halstead, KS

Boston, MA

Houston, TX

Vienna, Austria

New York, $N Y$

Ottawa, Canada

London, Canada

Paris, France

Rochester, MN

Salt Lake City, UT

Tucson, AZ

Farmington, $C T$

Rhône-Alpes, France

Brussels, Belgium

Coral Gables, FL

Oxford, United Kingdom

Fairfax, VA

Iowa City, IA

Boston, MA

Ann Arbor, MI

Denver, $C O$

Uppsala, Sweden

Bologna, Italy

Charleston, SC

Minneapolis, MN

Houston, TX

Nashville, TN

New York, NY

Sungnam, Republic of Korea

Seoul, Republic of Korea

Winnipeg, Canada

New York, NY

Seattle, WA

Baltimore, $M D$

Bergen, Norway

Zürich, Switzerland

Stanford, CA

Baltimore, $M D$

Bronx, NY

Cleveland, $\mathrm{OH}$

Chicago, IL

Rochester, MN

Fort Lauderdale, FL

New York, NY

Memphis, TN 


\begin{tabular}{|c|c|c|c|}
\hline Gregory Lewis & Hershey, PA & Stephen Lyman & New York, $N Y$ \\
\hline James Lewis & Philadelphia, PA & John Lynch & Seattle, WA \\
\hline Peter Lewis & Adelaide, Australia & Joseph Lynch & San Diego, CA \\
\hline Kirsten Leyland & Oxford, United Kingdom & Roger Lyon & Milwaukee, WI \\
\hline Sang-Hoon Lhee & Seoul, Republic of Korea & Zhen-Sheng Ma & Xi'an Shaanxi, China \\
\hline Bingyun Li & Morgantown, WV & C. Benjamin Ma & San Francisco, $C A$ \\
\hline Gertrude Yingyu Li & Ann Arbor, MI & Travis Maak & Salt Lake City, UT \\
\hline Guoan Li & Boston, MA & Jay Mabrey & Dallas, $T X$ \\
\hline Kung-Chia Li & Chiayi, Taiwan & William Macaulay & New York, $N Y$ \\
\hline Stephen Li & Palm Harbor, FL & Jason Machan & Providence, $R I$ \\
\hline Xudong Li & Charlottesville, VA & Rami Madanat & Boston, MA \\
\hline Yimei Li & Philadelphia, PA & Kim Madden & Hamilton, Canada \\
\hline Zhongyu Li & Winston-Salem, NC & Nicola Maffulli & London, United Kingdom \\
\hline Chen-Kun Liaw & Taipei, Taiwan & Robert Magnussen & Columbus, $\mathrm{OH}$ \\
\hline Alexander Liddle & Oxford, United Kingdom & Petra Magosch & Heidelberg, Germany \\
\hline Richard Lieber & La Jolla, CA & Aditya Maheshwari & Brooklyn, NY \\
\hline Meir Liebergall & Jerusalem, Israel & Ormonde Mahoney & Athens, GA \\
\hline Jay Lieberman & Los Angeles, CA & Radhashree Maitra & Bronx, $N Y$ \\
\hline Young Wook Lim & Seoul, Republic of Korea & Robert Majeska & New York, $N Y$ \\
\hline Noppachart Limpaphayom & Bangkok, Thailand & Keijo Mäkelä & Turku, Finland \\
\hline Orr Limpisvasti & Los Angeles, CA & Charles Malemud & Cleveland, $\mathrm{OH}$ \\
\hline Jinn Lin & Taipei, Taiwan & Mohammad Malik & Manchester, United Kingdom \\
\hline Patrick Lin & Houston, $T X$ & Theodore Malinin & Miami, $F L$ \\
\hline Sheldon Lin & Newark, NJ & Konstantinos Malizos & Larissa, Greece \\
\hline Thomas Lindenfeld & Cincinnati, $\mathrm{OH}$ & Mitchell Maltenfort & Philadelphia, PA \\
\hline Peter Lindgren & Stockholm, Sweden & Augustine Manadan & Chicago, IL \\
\hline Ronald Lindsey & Galveston, $T X$ & Laxmaiah Manchikanti & Paducah, $K Y$ \\
\hline Joseph Lipman & New York, $N Y$ & Sebastian Manegold & Berlin, Germany \\
\hline Katrin Lips & Giessen, Germany & Marco Manfrini & Bologna, Italy \\
\hline Heinz Lohrer & Frankfurt, Germany & Michael Manley & Wyckoff, NJ \\
\hline Adolph Lombardi & New Albany, $\mathrm{OH}$ & Kenneth Mann & Syracuse, $N Y$ \\
\hline Jason Long & West Chester, PA & Hans Manner & Zürich, Switzerland \\
\hline Mandi Lopez & Baton Rouge, LA & Sakthivel R. R. Manoharan & Birmingham, $A L$ \\
\hline Mattia Loppini & Rome, Italy & Mark Manoso & Tacoma, WA \\
\hline Stephan Lorenz & Munich, Germany & M.M. Manring & Columbus, $\mathrm{OH}$ \\
\hline Scott Lovald & Menlo Park, CA & Carlos Mantilla & Rochester, $M N$ \\
\hline Giovanni Lovisetti & Menaggio, Italy & Alfonso Manzotti & Milan, Italy \\
\hline David Lowenberg & Redwood City, CA & Hilal Maradit Kremers & Rochester, $M N$ \\
\hline Santiago Lozano-Calderón & Boston, $M A$ & Luis Marchi & São Paulo, Brazil \\
\hline John Lubahn & Erie, $P A$ & Camelia Marculescu & Charleston, SC \\
\hline John Lubicky & Morgantown, $W V$ & Randall Marcus & Cleveland, $\mathrm{OH}$ \\
\hline George Lucas & Wichita, KS & Sven Märdian & Berlin, Germany \\
\hline James Luck & Los Angeles, $C A$ & Rodrigo Mardones & Santiago, Chile \\
\hline Austin Lugar & Rosemont, IL & Danica Marinac-Dabic & Silver Spring, $M D$ \\
\hline Tun Hing Lui & Hong Kong, China & David Markel & Southfield, MI \\
\hline Keith Luk & Hong Kong, China & Adam Marmon & Newark, DE \\
\hline David Lunardini & South Burlington, VT & Carlos Marques & Hamburg, Germany \\
\hline Göran Lundborg & Malmö, Sweden & Guido Marra & Chicago, IL \\
\hline Douglas Lundy & Marietta, GA & Jill Marsteller & Baltimore, $M A$ \\
\hline David Lutton & New York, $N Y$ & John Martell & Chicago, IL \\
\hline Marios Lykissas & Ioannina, Greece & David Martin & Chapel Hill, NC \\
\hline
\end{tabular}


Hal Martin

J. Bohannon Mason

Bassam Masri

Koichi Masuda

Fabrizio Matassi

Frederick Matsen, III

Takao Matsubara

Dean Matsuda

Shuichi Matsuda

Larry Matthews

Paul Matuszewski

Blaž Mavčič

Marius Mayerhoefer

Daniel Mazanec

Augustus Mazzocca

Daniel Mazzucco

James McAuley

G. Grady McBride

Richard McCalden

L. Pearce McCarty, III

Eugene McCloskey

George McCluskey

Jenny McConnell

John McDonald

Matthew McElvany

Edward McFarland

Richard McGough

Desirae McKee

Harry McKellop

Ryan McLemore

Amy McNulty

Margaret McNulty

Simon Mears

Giovanna Medina

John Meding

R.M. Dominic Meek

Geert Meermans

Reinhard Meier

Gerd Melkus

Claudio Mella

Menachem Meller

Stavros Memtsoudis

Christopher Mendias

Lawrence Menendez

Kevin Mennitt

Addisu Mesfin

Terry Messer

Dominik Meyer

James Michelson

Rolf Miehlke

Henri Migaud
Dallas, TX

Charlottesville, NC

Vancouver, Canada

La Jolla, CA

Florence, Italy

Seattle, WA

Mie, Japan

Los Angeles, CA

Kyoto, Japan

Ann Arbor, MI

Baltimore, MD

Ljubljana, Slovenia

Vienna, Austria

Cleveland, $\mathrm{OH}$

Farmington, $C T$

Philadelphia, PA

London, Canada

Orlando, FL

London, Canada

Edina, MN

Sheffield, United Kingdom

Columbus, $\mathrm{OH}$

Mosman, Australia

Reno, NV

Santa Rosa, CA

Baltimore, $M D$

Pittsburgh, PA

Lubbock, TX

Los Angeles, CA

Frederick, $M D$

Durham, NC

Chicago, IL

Little Rock, AR

Sao Paulo, Brazil

Mooresville, IN

Glasgow, United Kingdom

Bergen Op Zoom, Netherlands

Meiningen, Germany

Ottawa, Canada

Santiago, Chile

Philadelphia, PA

New York, NY

Ann Arbor, MI

Los Angeles, CA

New York, $N Y$

Rochester, NY

Raleigh, NC

Zürich, Switzerland

Burlington, VT

Wiesbaden, Germany

Lille, France
Mark Mighell

Lone Mikkelsen

Neal Millar

Anna Miller

Benjamin Miller

Freeman Miller

Mark Miller

Theodore Miller

Ingrid Milosev

Joe Minchew

Anthony Miniaci

Yukihide Minoda

Hermes Miozzari

Thomas Mittlmeier

Mark Mizel

Hitesh Modi

Todd Moen

Khalid Mohammad

David Mohler

Peter Moley

Fergal Monsell

Robert Monsey

Michael Mont

Corey Montgomery

Bryan Moon

Seong-Hwan Moon

K. David Moore

Matthew Moran

Lars Morawietz

Alexandre Moreau-Gaudry

David Morgenroth

Fintan Moriarty

Takeshi Morii

Hisao Moritomo

Michael Morlock

Philipp Moroder

Brent Morris

Dylan Morrissey

Michael Moskal

Ronald Moskovich

Penny Moss

Caroline Mouton

Christopher Mow

Xiaodong $\mathrm{Mu}$

Scott Mubarak

Lindsay Muir

Kevin Mulhall

Michiel Mulier

Arun Mullaji

Yue Wai Mun

Stephen Munns
Temple Terrace, FL

Silkeborg, Denmark

Glasgow, United Kingdom

St. Louis, MO

Iowa City, IA

Wilmington, $D E$

Pittsburgh, PA

New York, NY

Ankaran, Slovenia

Durham, NC

Cleveland, $\mathrm{OH}$

Osaka, Japan

Geneva, Switzerland

Rostock, Germany

Miami, FL

Seoul, Republic of Korea

Dallas, TX

Indianapolis, IN

Redwood City, CA

New York, $N Y$

Bristol, United Kingdom

Williston, $V T$

Cleveland, $\mathrm{OH}$

Little Rock, AR

Houston, TX

Seoul, Republic of Korea

Birmingham, AL

Fairfield, CT

Berlin, Germany

La Tronche, France

Seattle, WA

Davos, Switzerland

Mitaka, Tokyo, Japan

Osaka, Japan

Hamburg, Germany

Salzburg, Austria

Lexington, $K Y$

London, United Kingdom

Sellersburg, IN

New York, NY

Perth, Australia

Luxembourg, Luxembourg

Pinole, $C A$

Pittsburgh, PA

San Diego, CA

Salford, United Kingdom

Dublin, Ireland

Lubbeek, Belgium

Mumbai, India

Singapore, Singapore

Kansas City, KS 
Takanori Murakami

Tsuyoshi Murase

James Murphy

Stephen Murphy

James Murray

Peter Murray

George Muschler

D. Luis Muscolo

Jonathan Mutimer

Mark Myerson

Young Gon $\mathrm{Na}$

Nash Naam

Jan Nadorf

Sandesh C. S. Nagamani

Hideki Nagashima

Markus Nagl

Satoshi Nagoya

Takeo Nagura

Ladislav Nagy

Koichi Nakagawa

Eiichiro Nakamura

Norimasa Nakamura

Takashi Nakamura

Toshiyasu Nakamura

Yasuharu Nakashima

Denis Nam

Robert Namba

Surena Namdari

Sumon Nandi

George Nanos

Amir Ali Narvani

Ahmad Nassr

Tassos Natsakis

Douglas Naudie

Levon Nazarian

Manfred Nelitz

Charles Nelson

Sandra Nelson

Szilard Nemes

Jeffrey Nepple

Valentin Neuhaus

Daniëlle Neut

Peter Newton

Kwan-Ching Ng

Uyen-Sa Nguyen

Christophe Nich

Claude Nichols, III

Jun Nishida

Yoshihiro Nishida

Takashi Nishii

Raimo Niskanen
Sapporo, Japan

Osaka, Japan

Philadelphia, PA

Boston, MA

Bristol, United Kingdom

Jacksonville, $F L$

Cleveland, $\mathrm{OH}$

Buenos Aires, Argentina

Cheltenham, United Kingdom

Baltimore, $M D$

Gyeonggi-do, Republic of Korea

Effingham, IL

Heidelberg, Germany

Houston, TX

Yonago, Japan

Innsbruck, Austria

Sapporo, Japan

Tokyo, Japan

Zürich, Switzerland

Sakura, Japan

Fukuoka, Japan

Osaka, Japan

Kyoto, Japan

Tokyo, Japan

Fukuoka, Japan

Chicago, IL

Irvine, $C A$

Philadelphia, PA

Toledo, $\mathrm{OH}$

Bethesda, MD

London, United Kingdom

Rochester, MN

Cluj Napoca, Romania

London, Canada

Philadelphia, PA

Ulm, Germany

Philadelphia, PA

Boston, MA

Gothenburg, Sweden

St. Louis, MO

Zürich, Switzerland

Groningen, Netherlands

San Diego, CA

London, United Kingdom

Worchester, MA

Garches, France

Burlington, $V T$

Morioka, Japan

Nagoya, Japan

Osaka, Japan

Lahti, Finland
Carl Nissen

Alan Nixon

Christopher Niyibizi

Rémy Nizard

Philip Noble

Michael Nogler

Monica Nogueira

Nicolas Noiseux

Syed Noor

Laurent Nove-Josserand

Wendy Novicoff

Gordon Nuber

Pierce Nunley

Jeffry Nyman

Lukas Nystrom

Daniel O'Connor

Mary O'Connor

Richard O'Donnell

Turlough O'Donnell

Tadhg O'Gara

Darrell Ogilvie-Harris

Joo Han Oh

Ken Okazaki

Steven Olson

Reza Omid

David Ong

Kevin Ong

William Oppenheim

Lori Orlando

Justin Orr

Shunzo Osaka

Daryl Osbahr

Daniel Osei

Greg Osgood

A Lee Osterman

Tye Ouzounian

Volkan Oztuna

Maurizio Pacifici

Douglas Padgett

Geert Pagenstert

Mark Pagnano

Paolo Paladini

Jeya Palan

Robert Palisano

Henrik Palm

Vinod Panchbhavi

Dilbans Pandher

Panayiotis Papagelopoulos

Richard Papandrea

Jin-Young Park

Min Jong Park
Farmington, $C T$

Ithica, $N Y$

Hershey, $P A$

Paris, France

Houston, TX

Innsbruck, Austria

São Paulo, Brazil

Iowa City, IA

Karachi, Pakistan

Lyon, France

Charlottesville, VA

Chicago, IL

Shreveport, $L A$

Nashville, TN

Maywood, IL

Houston, TX

New Haven, CT

San Francisco, $C A$

Dublin, Ireland

Lynbrook, NY

Toronto, Canada

Gyeonggi-do, Republic of Korea

Fukuoka, Japan

Durham, NC

Los Angeles, $C A$

Wellington, New Zealand

Philadelphia, PA

Los Angeles, CA

Durham, NC

El Paso, TX

Tokyo, Japan

Baltimore, MD

St. Louis, MO

Baltimore, $M D$

Philadelphia, PA

Tarzana, CA

Mersin, Turkey

Philadelphia, PA

New York, NY

Basel, Switzerland

Rochester, MN

Cattolica, Italy

Leicester, United Kingdom

Philadelphia, PA

Boston, MA

Galveston, TX

Jalandhar, India

Athens, Greece

Waukesha, WI

Seoul, Republic of Korea

Seoul, Republic of Korea 
Kyung Hyun Park-Min

Michael Parks

Sébastien Parratte

William Parrish

Moby Parsons

Theodore Parsons

Eleftherios Paschalis

Gail Pashos

Alpesh Patel

Robin Paton

Bishnu Prasad Patro

Joshua Patt

Francis Patterson

Helene Pavlov

E. Scott Paxton

Elizabeth Paxton

Christopher Peach

Michael Pearl

Adam Pearson

Christopher Pelt

Giuseppe Peretti

Carsten Perka

Anthony Perruccio

Anthony Petrella

Frank Petrigliano

Tilman Pfitzner

Marc Philippon

Frank Phillips

Wesley Phipatanakul

Frédéric Picard

Piero Picci

David Pichora

David Pienkowski

Jeffery Pierson

Luiz Pimenta

Michael Pinzur

Shafique Pirani

Miguel Pirela-Cruz

Rocco Pitto

Nicolas Piuzzi

Peter Pizzutillo

Anna Plaas

Christian Plaass

Anton Plakseychuk

Hanns Plenk

Avraam Ploumis

David Podeszwa

Kornelis Poelstra

Stéphane Poitras

Enrico Pola

Elizabeth Polfer
New York, NY

New York, $N Y$

Marseille, France

Lancaster, PA

Somersworth, $\mathrm{NH}$

Detroit, MI

Vienna, Austria

St. Louis, MO

Salt Lake City, UT

Blackburn, United Kingdom

Bhubaneswar, India

Charlotte, NC

Newark, NJ

New York, NY

Providence, RI

El Cajon, CA

Manchester, United Kingdom

Los Angeles, CA

Lebanon, $\mathrm{NH}$

Salt Lake City, UT

Milan, Italy

Berlin, Germany

Toronto, Canada

Golden, $\mathrm{CO}$

Los Angeles, CA

Berlin, Germany

Vail, $C O$

Chicago, IL

Loma Linda, CA

Clydebank, United Kingdom

Bologna, Italy

Kingston, Canada

Lexington, $K Y$

Indianapolis, IN

São Paulo, Brazil

Maywood, IL

New Westminster, Canada

El Paso, TX

Auckland, New Zealand

Cleveland, $\mathrm{OH}$

Philadelphia, PA

Chicago, IL

Hannover, Germany

Pittsburgh, PA

Vienna, Austria

Ioannina, Greece

Dallas, TX

Destin, FL

Ottawa, Canada

Rome, Italy

Bethesda, MD
Sandra Poliachik

John Polousky

Hans Polzer

John Popovich, Jr.

Raphaël Porcher

María Portillo

Zachary Post

Benjamin Potter

Paul Pottinger

Bruno Pozzetto

Kodali Prasad

Andreas Prescher

Elisabeth Presterl

Mark Price

Rhonda Prisby

Wolfgang Prodinger

Themistocles Protopsaltis

Thomas Pufe

Andrew Pugely

Ari-Pekka Puhto

Shannon Puloski

James Purtill

Sophie Putman

Carlo Quattrocchi

Robin Queen

Patricia Quinlan

Sheeraz Qureshi

Fernando Radice

Christof Radler

M. Farooq Rai

Steven Raikin

Rajiv Rajani

Kawan Rakhra

Stuart Ralston

Gordon Ramage

Shalini Ramasunder

Maximiliano Ranalletta

Anil Ranawat

R. Lor Randall

Ellen Raney

Allison Rao

Bhaskar Rao

Nalini Rao

Timothy Rapp

Michael Raschke

Lars Rasmussen

Karl Rathjen

Parthiv Rathod

John Ratliff

Michael Rauh

Robert Raw
Seattle, WA

Centennial, $\mathrm{CO}$

Munich, Germany

East Lansing, MI

Paris, France

El Prat de Llobregat, Spain

Philadelphia, PA

Bethesda, MD

Seattle, WA

Saint-Etienne, France

Merthyr Tydfil, United Kingdom

Aachen, Germany

Vienna, Austria

Worcester, MA

Arlington, TX

Innsbruck, Austria

New York, NY

Aachen, Germany

Iowa City, IA

Oulu, Finland

Calgary, Canada

Philadelphia, PA

Lille Cedex, France

Rome, Italy

Blacksburg, VA

New York, $N Y$

New York, $N Y$

Santiago, Chile

Vienna, Austria

St. Louis, $M O$

Philadelphia, PA

San Antonio, TX

Ottawa, Canada

Edinburgh, United Kingdom

Glasgow, United Kingdom

Durham, NC

Buenos Aires, Argentina

New York, NY

Salt Lake City, UT

Honolulu, HI

Stanford, $C A$

Memphis, TN

Pittsburgh, PA

New York, $N Y$

Münster, Germany

Copenhagen, Denmark

Dallas, TX

New York, $N Y$

Stanford, CA

Orchard Park, NY

Coralville, IA 


\begin{tabular}{|c|c|c|c|}
\hline John Ready & Boston, MA & James Ross & St. Louis, $M O$ \\
\hline Glenn Rechtine & Bay Pines, FL & Roberto Rossi & Turin, Italy \\
\hline Sven Reese & Munich, Germany & Dominique Rouleau & Montreal, Canada \\
\hline Glenn Regehr & Vancouver, Canada & Dennis Roy & Portland, OR \\
\hline Ian Reid & Grafton, New Zealand & S. Robert Rozbruch & New York, $N Y$ \\
\hline J. Spence Reid & Hershey, $P A$ & Tamara Rozental & Boston, MA \\
\hline Olav Reikeras & Oslo, Norway & Harry Rubash & Boston, MA \\
\hline Donald Reilly & Brighton, $M A$ & David Ruch & Winston-Salem, NC \\
\hline Kent Reinker & San Antonio, $T X$ & Hannes Rudiger & Zürich, Switzerland \\
\hline John Reith & Gainesville, $F L$ & Luke Rudmik & Calgary, Canada \\
\hline Charles Reitman & Charleston, SC & Johannes Rueger & Hamburg, Germany \\
\hline Daniel Resnick & Madison, WI & Pietro Ruggieri & Padova, Italy \\
\hline Eric Ricchetti & Cleveland, $\mathrm{OH}$ & Miguel Ruiz-Iban & Madrid, Spain \\
\hline William Ricci & New York, $N Y$ & Alessandro Russo & Bologna, Italy \\
\hline Benjamin Ricciardi & New York, NY & Sanjeev Sabharwal & Newark, NJ \\
\hline David Rice & Auckland, New Zealand & Marlis Sabo & Calgary, Canada \\
\hline Heather Richard & Dallas, $T X$ & Gregory Saboeiro & New York, $N Y$ \\
\hline Philippe Richebé & Montreal, Canada & Robert Sah & La Jolla, CA \\
\hline Bradford Richmond & Cleveland, $\mathrm{OH}$ & Jose Salazar-Torres & Belfast, United Kingdom \\
\hline Mark Rickman & Adelaide, Australia & Khaled Salem & Cairo, Egypt \\
\hline Daniel Riddle & Richmond, VA & Cassandra Salgado & Charleston, SC \\
\hline Michael Ries & Carson City, NV & Erdjan Salih & Boston, MA \\
\hline Scott Riester & Rochester, MN & Robert Salk & San Francisco, $C A$ \\
\hline K. Daniel Riew & St. Louis, $M O$ & Sari Salminen & Trondheim, Norway \\
\hline Wim Rijnen & Nijmegen, Netherlands & Gretchen Salsich & St. Louis, $M O$ \\
\hline Lee Riley & Baltimore, $M D$ & Charles Saltzman & Salt Lake City, UT \\
\hline David Ring & Austin, TX & Matthew Saltzman & Chicago, IL \\
\hline Lawrence Rinsky & Stanford, $C A$ & James Sanders & Rochester, NY \\
\hline Makarand Risbud & Philadelphia, PA & Wudbhav Sankar & Philadelphia, PA \\
\hline Damian Rispoli & York, $P A$ & Laura Santambrogio & New York, $N Y$ \\
\hline William Robb & Glenview, IL & Edward Santos & Minneapolis, $M N$ \\
\hline Mark Robbin & Cleveland, $\mathrm{OH}$ & Neil Saran & Montreal, Canada \\
\hline Craig Roberts & Louisville, $K Y$ & Robert Satcher & Houston, TX \\
\hline William Robertson, Jr. & Bethesda, MD & Lucia Savarino & Bologna, Italy \\
\hline Scott Rodeo & New York, $N Y$ & Olga Savvidou & Agia Paraskevi, Greece \\
\hline William Rodkey & Vail, $C O$ & Chigusa Sawamura & Tokyo, Japan \\
\hline Jose Rodriguez & New York, $N Y$ & Mark Scarborough & Gainesville, $F L$ \\
\hline Benedict Rogers & Toronto, Canada & Michael Schafer & Chicago, IL \\
\hline Jeffrey Roh & Kirkland, WA & Jonathan Schaffer & Cleveland, $\mathrm{OH}$ \\
\hline Young Hak Roh & Seoul, Republic of Korea & Hanns-Peter Scharf & Mannheim, Germany \\
\hline Stephan Röhrl & Oslo, Norway & Thomas Scharschmidt & Columbus, $\mathrm{OH}$ \\
\hline Magne Røkkum & Oslo, Norway & Robert Schenck & Albuquerque, NM \\
\hline Maarten Röling & Delft, Netherlands & Gereon Schiffer & Bergisch Gladbach, Germany \\
\hline Carlo Romano & Milano, Italy & Daniel Schlatterer & Atlanta, $G A$ \\
\hline Pol Rommens & Mainz, Germany & Thomas Schmalzried & Los Angeles, $C A$ \\
\hline Andreas Roposch & London, United Kingdom & Andrew Schmidt & Plymouth, $M N$ \\
\hline Peter Rose & Rochester, MN & Steven Schmitt & Cleveland, $\mathrm{OH}$ \\
\hline Dieter Rosenbaum & Muenster, Germany & Robert Schneider & New York, $N Y$ \\
\hline Björn Rosengren & Malmo, Sweden & Christoph Schnurr & Viersen, Germany \\
\hline Ann Rosenthal & Milwaukee, WI & Perry Schoenecker & St. Louis, $M O$ \\
\hline Howard Rosenthal & Overland Park, $K S$ & Matti Scholz & Frankfurt, Germany \\
\hline
\end{tabular}


Timothy Schrader

B. Willem Schreurs

Mark Schrumpf

Michael Schuetz

Joseph Schwab

Mitchell Schwaber

Adam Schwartz

Edward Schwarz

Ran Schwarzkopf

Richard Schwend

Marcus Sciadini

Joseph Sclafani

Alexander Scott

Celeste Scotti

Hazel Screen

Thomas Sculco

Sean Scully

Laurent Sedel

Lee Segal

Neil Segal

Cheryle Seguin

Matthew Seidel

Jon Sekiya

David Seligson

Parham Sendi

Alpaslan Senkoylu

Brian Sennett

Manish Sethi

Mathew Sewell

Nikolaos Sferopoulos

Apurva Shah

Sameer Shah

A. Nick Shamie

Hala Shamsuddin

Jesse Slade Shantz

Peter Sharkey

Adrija Sharma

Vivek Sharma

Nigel Sharrock

Brian Shaw

Kevin Shea

Jason Shearn

Eoin Sheehan

K. Donald Shelbourne

Neil Sheth

Derek Shia

Steven Shin

Hiromu Shoji

Won Yong Shon

Benjamin Shore

Nigel Shrive
Atlanta, GA

Nijmegen, Netherlands

San Francisco, CA

Kelvin Grove, Australia

Boston, MA

Tel-Aviv, Israel

Phoenix, AZ

Rochester, NY

New Rochelle, $N Y$

Albuquerque, NM

Baltimore, $M D$

San Diego, CA

Vancouver, Canada

Milano, Italy

London, United Kingdom

New York, $N Y$

Jacksonville, $N C$

Paris, France

Madison, WI

Iowa City, IA

London, Canada

Phoenix, AZ

Ann Arbor, MI

Louisville, $K Y$

Liestal, Switzerland

Ankara, Turkey

Philadelphia, PA

Nashville, TN

Middlesbrough, United Kingdom

Thessaloniki, Greece

Iowa City, IA

San Diego, CA

Santa Monica, CA

Silver Spring, $M D$

San Francisco, CA

Philadelphia, PA

Knoxville, TN

Minneapolis, $M N$

New York, NY

Colorado Springs, $\mathrm{CO}$

Boise, ID

Cincinnati, $\mathrm{OH}$

Tullamore, Ireland

Indianapolis, IN

Philadelphia, PA

New Haven, CT

Los Angeles, CA

Riverside, $C A$

Seoul, Republic of Korea

Boston, MA

Calgary, Canada
Beth Shubin-Stein

Klaus-Arno Siebenrock

Daniel Siegal

Jodi Siegel

Sorin Siegler

Rafael Sierra

Harri Sievänen

Alla Sikorskii

Craig Silverton

Franklin Sim

Shawn Simmons

Conrad Simpfendorfer

David Sinacore

Marc Sinclair

Kern Singh

Robert Siston

Bjorn Skallerud

Harry Skinner

John Skinner

Antigone Skopelitou

Keith Skubitz

Eerik Skyttä

James Slauterbeck

James Slover

Kathleen A. Sluka

Gaston Slullitel

Dale Smith

Eric Smith

Peter Smith

Timothy So

Carolyn Sofka

David Sohn

Lucian Solomon

Kit Song

Kyung-Jin Song

Sang Jun Song

Nelson SooHoo

Alex Soriano

Mark Spangehl

Lawrence Specht

John Sperling

David Spiegel

Robert Spinner

Steven Spires

Andrew Spitzer

Paul Sponseller

Bryan Springer

Jan Spyridakis

Matthew Squire

Shafic Sraj

Rita Stagni
New York, NY

Bern, Switzerland

Detroit, MI

Worcester, MA

Philadelphia, PA

Rochester, MN

Tampere, Finland

East Lansing, MI

Detroit, MI

Rochester, MN

Iowa City, IA

Cleveland, $\mathrm{OH}$

St. Louis, MO

Dubai, United Arab Emirates

Chicago, IL

Columbus, $\mathrm{OH}$

Trondheim, Norway

Fullerton, $C A$

Stanmore, United Kingdom

Arta, Greece

Minneapolis, MN

Tampere, Finland

Burlington, VT

New York, $N Y$

Iowa City, IA

Rosario, Argentina

Bethesda, MD

Boston, MA

Chicago, IL

Hong Kong, China

New York, $N Y$

Toledo, $\mathrm{OH}$

Adelaide, Australia

Seattle, WA

Jeonju, Republic of Korea

Seoul, Republic of Korea

Los Angeles, $C A$

Barcelona, Spain

Phoenix, AZ

Burlington, $M A$

Rochester, MN

Philadelphia, PA

Rochester, MN

Nashville, TN

Los Angeles, CA

Baltimore, $M D$

Charlotte, NC

Seattle, WA

Madison, WI

Weston, WV

Bologna, Italy 


\begin{tabular}{|c|c|c|c|}
\hline Jeffrey B. Stambough & St. Louis, MO & Benjamin Taylor & Columbus, $\mathrm{OH}$ \\
\hline Jeffery L. Stambough & Cincinnati, $\mathrm{OH}$ & Matthew Teeter & London, Canada \\
\hline Christopher Standaert & Seattle, WA & Robert Teitge & Detroit, $M I$ \\
\hline Andre Stark & Stockholm, Sweden & Sabine Tejpar & Leuven, Belgium \\
\hline Peter Stasikelis & Greenville, $S C$ & Nirmal Tejwani & New York, $N Y$ \\
\hline Roland Staud & Berlin, Germany & Johnna Temenoff & Atlanta, $G A$ \\
\hline Anna Stefánsdóttir & Lund, Sweden & Hidetomi Terai & Osaka, Japan \\
\hline Marla Steinbeck & Philadelphia, PA & Richard Terek & Providence, $R I$ \\
\hline Ely Steinberg & Tel-Aviv, Israel & Kevin Tetsworth & Brisbane, Australia \\
\hline Dirk Stengel & Berlin, Germany & Teun Teunis & Utrecht, Netherlands \\
\hline Simon Steppacher & Bern, Switzerland & David Teuscher & Beaumont, $T X$ \\
\hline Steven Stern & Northfield, IL & Aysen Tezcaner & Ankara, Turkey \\
\hline Martin Stevens & Groningen, Netherlands & T.N. Theologis & Headington, United Kingdom \\
\hline James Stiehl & Centralia, $I L$ & Lars Theyse & Utrecht, Netherlands \\
\hline Kevin Stone & San Francisco, $C A$ & Emmanuel Thienpont & Brussels, Belgium \\
\hline Paul Stoodley & Columbus, $\mathrm{OH}$ & Geb Thomas & Iowa City, IA \\
\hline Geert Streekstra & Amsterdam, Netherlands & Geraint Thomas & Oxford, United Kingdom \\
\hline Marcus Streit & Heidelberg, Germany & John Thomas & Morgantown, $W V$ \\
\hline Peter Strouse & Ann Arbor, MI & Stephen Thomas & Philadelphia, PA \\
\hline Steven Stuchin & New York, $N Y$ & George Thompson & Davis, $C A$ \\
\hline Ottokar Stundner & Salzburg, Austria & George H. Thompson & Cleveland, $\mathrm{OH}$ \\
\hline Daniel Sturnick & New York, $N Y$ & Jeffrey Thomson & Hartford, $C T$ \\
\hline Edwin $\mathrm{Su}$ & New York, $N Y$ & Thomas Thornhill & Boston, MA \\
\hline Arnold Suda & Mannheim, Germany & Jennifer Tilley & Notre Dame, IN \\
\hline Nobuhiko Sugano & Osaka, Japan & Beverlie Ting & Boston, MA \\
\hline Dale Sumner & Chicago, IL & Scott Tintle & Bethesda, MD \\
\hline Yu-Long Sun & Shenzhen, China & Fotios Tjoumakaris & Egg Harbor Township, NJ \\
\hline Martin Sundberg & Malmö, Sweden & E. Bruce Toby & Kansas City, KS \\
\hline Martin Svehlik & Graz, Austria & Silvio Tocco & Parma, Italy \\
\hline Vineeta Swaroop & Chicago, IL & Eric Tolo & Peabody, MA \\
\hline Bart Swierstra & Nijmegen, Netherlands & Masuhiro Tomita & Osaka, Japan \\
\hline Robert Szabo & Sacramento, $C A$ & Jérôme Tonetti & Cedex, France \\
\hline Elizabeth Szalay & Albuquerque, NM & John Tongue & Portland, OR \\
\hline Timothy Taft & Chapel Hill, NC & Brian Toolan & Chicago, IL \\
\hline Shinro Takai & Tokyo, Japan & Michael Torchia & Rochester, $M N$ \\
\hline Carl Talmo & Boston, $M A$ & Paul Tornetta, III & Boston, MA \\
\hline Kazuya Tamai & Tochigi, Japan & Armando Torres-Gomez & Mexico City, Mexico \\
\hline Robert Tamurian & Sacramento, $C A$ & Laura Tosi & Washington, $D C$ \\
\hline Virak Tan & Newark, NJ & Peter Trafton & Providence, $R I$ \\
\hline Pedro Tanaka & Stanford, $C A$ & Anders Troelsen & Hvidovre, Denmark \\
\hline Shinya Tanaka & Iruma-gun, Japan & Yao-Hung Tsai & Putz City, Taiwan \\
\hline Aaron Tande & Rochester, MN & Yusuke Tsuda & Tokyo, Japan \\
\hline Jin Bo Tang & Providence, $R I$ & Dean Tsukayama & Minneapolis, $M N$ \\
\hline Xiaodong Tang & Beijing, China & Per-Ulf Tunn & Berlin, Germany \\
\hline Boonsin Tangtrakulwanich & Haadyai, Thailand & Dennis Turk & Seattle, WA \\
\hline Michael Tanzer & Montreal, Canada & Bruce Twaddle & Auckland, New Zealand \\
\hline John Tarlton & Langford, United Kingdom & Koray Unay & Istanbul, Turkey \\
\hline Yasutaka Tashiro & Pittsburgh, PA & Frank Underwood & Evansville, IN \\
\hline Robert Tashjian & Salt Lake City, UT & Aasis Unnanuntana & Bangkok, Thailand \\
\hline Mark Tauber & Munich, Germany & Vidyadhar Upasani & San Diego, $C A$ \\
\hline Michael Taunton & Rochester, MN & Dietmar Urbach & Magdeburg, Germany \\
\hline
\end{tabular}


Robert Urban

James Urbaniak

Juan Uribe

Vijay Vad

Rahul Vaidya

Thomas Vail

Carolina Valencia

Heather Vallier

Harold van Bosse

Douglas Van Citters

Michiel van de Sande

Lennard van den Boom

Frans van der Helm

Catherine Van Der Straeten

Marnix van Holsbeeck

Esther van Lieshout

Roger van Riet

Liza van Steenbergen

Job Van Susante

Alexander Van Tongel

Wes Van Voorhis

Daniel Vanel

Manish Varshney

Sonia Vasdev

Haris Vasiliadis

Patrick Vavken

Christian Veillette

Job Velazquez Moreno

Pascal-André Vendittoli

Jan Verhaar

Michael Verhofstad

Raphael Vialle

Jan Victor

Brian Victoroff

Vincent Vigorita

Marta Villarraga

Kelly Vince

Heather Vincent

Abinash Virk

Markus Vogt

Christine Voigt

David Volgas

Rebecca von der Heyde

Rüdiger von Eisenhart-Rothe

Marius von Knoch

Thord von Schewelov

Herbert von Schroeder

Michael Voor

James Waddell

Eugene Wai

Monique Walenkamp
Chicago, IL

Durham, NC

Tampa, FL

New York, $N Y$

Detroit, MI

San Francisco, $C A$

Terre Haute, IN

Cleveland, $\mathrm{OH}$

Philadelphia, PA

Hanover, $\mathrm{NH}$

Leiden, Netherlands

Groningen, Netherlands

Delft, Netherlands

Ghent, Belgium

Detroit, MI

Rotterdam, Netherlands

Edegem, Belgium

Eindhoven, Netherlands

Arnhem, Netherlands

Ghent, Belgium

Seattle, WA

Bologna, Italy

Jamshedpur, India

Springfield, IL

Ioannina, Greece

Boston, MA

Toronto, Canada

México City, Mexico

Montreal, Canada

Rotterdam, Netherlands

Rotterdam, Netherlands

Paris, France

Gent, Belgium

Cleveland, $\mathrm{OH}$

Brooklyn, NY

Philadelphia, PA

Newtown, New Zealand

Gainesville, $F L$

Rochester, MN

Baar, Switzerland

Hannover, Germany

Columbia, $\mathrm{MO}$

St. Louis, MO

Frankfurt, Germany

Bremerhaven, Germany

Malmö, Sweden

Toronto, Canada

Louisville, $K Y$

Toronto, Canada

Ottawa, Canada

Amsterdam, Netherlands
Peter Walker

Richard Walker

Lindley Wall

W. Angus Wallace

Henrik Wallander

William Walsh

Ching-Jen Wang

Feng-Sheng Wang

James Wang

Vincent Wang

Wenhai Wang

Yan Wang

Yisheng Wang

Tony Wanich

John Ware

Simon Warfield

Winston Warme

David Warwick

Daniel Wascher

Ray Wasielewski

Adam Watts

Chad Watts

Jennifer Wayne

Brian Weatherford

Michael Weaver

Stephen Weber

John Wedge

Yiyong Wei

Lars Weidenhielm

Douglas Weinberg

Bradley Weiner

Dennis Weiner

Scott Weiner

Paul Weinhold

Stuart Weinstein

Lawrence Weis

Arnold-Peter Weiss

Jennifer Weiss

Samuel Wellman

Joseph Wenke

Glenn Wera

Frederick Werner

F. Todd Wetzel

Peter Whang

James White

Neil White

Richard White

Daniel Whiting

Roger Widmann

Andreas Widmer

Shlomo Wientroub
New York, $N Y$

La Jolla, CA

St. Louis, $M O$

Nottingham, United Kingdom

Gefle, Sweden

Sydney, Australia

Kaosiung, Taiwan

Kaohsiung, Taiwan

Pittsburgh, PA

Blacksburg, VA

Philadelphia, PA

Beijing, China

Zhengzhou, China

Bronx, NY

Worcester, MA

Boston, MA

Seattle, WA

Southampton, United Kingdom

Albuquerque, NM

Columbus, $\mathrm{OH}$

Wigan, United Kingdom

Rochester, MN

Richmond, VA

Grand Rapids, MI

Boston, MA

Sacramento, $C A$

Toronto, Canada

Shanghai, China

Stockholm, Sweden

Cleveland, $\mathrm{OH}$

Hershey, PA

Akron, $\mathrm{OH}$

Akron, $\mathrm{OH}$

Chapel Hill, NC

Iowa City, IA

Georgetown, $C T$

Providence, RI

Los Angeles, CA

Durham, NC

Houston, TX

Cleveland, $\mathrm{OH}$

Syracuse, $N Y$

Philadelphia, PA

New Haven, CT

Charlottesville, VA

Calgary, Canada

Columbia, MO

Missoula, MT

New York, NY

Basel, Switzerland

Tel-Aviv, Israel 
Karl Wieser

Ross Wilkins

Shelley Wilkinson

Gerald Williams

James Williams

Mark Williams

Nicole Wilson

Markus Wimmer

Reinhard Windhager

Marcel Winkelmann

Eivind Wits $\emptyset$

Johan Witt

Angelique Witteveen

Felasfa Wodajo

Erik Wolf

Jennifer Wolf

Robert Wolf

David Wong

Hee-Kit Wong

Steven Woolson

Peter Worsley

James Wright

Patty Wright

Rick Wright

Jim Wu

Thomas Wuest

James Wylie

Pablo Yagupsky

Takuaki Yamamoto

Shigui Yan

Rong-Sen Yang

Sai-Wei Yang

Xinlin Yang

Jeffrey Yao

Piers Yates

Peter Wai-Pan Yau

David Yen
Zürich, Switzerland

Denver, CO

Brisbane, Australia

Philadelphia, PA

Chicago, IL

LaCrosse, WI

St. Louis, MO

Chicago, IL

Vienna, Austria

Hannover, Germany

Trondheim, Norway

London, United Kingdom

Amsterdam, Netherlands

Fairfax, VA

Bethesda, MD

Farmington, $C T$

Birmingham, $A L$

Denver, $C O$

Singapore, Singapore

Menlo Park, CA

Southampton, United Kingdom

Toronto, Canada

Nashville, TN

St. Louis, MO

Boston, MA

Eugene, OR

Farmington, CT

Beer-Sheva, Israel

Fukuoka, Japan

Hangzhou, China

Taipei, Taiwan

Taipei, Taiwan

Charlottesville, VA

Redwood City, CA

Crawley, Australia

Hong Kong, China

Kingston, Canada
Seng Jin Yeo

Christos Yiannakopoulos

Cengiz Yilmaz

Zhong-gang Yin

Yuichi Yoshii

James Youdas

David Young

Simon Young

Alastair Younger

Brandon Yuan

Varah Yuenyongviwat

Michael Zaccariello

Terri Zachos

Charalampos Zalavras

Edward Zaragoza

Boris Zelle

Alan Zhang

Weiya Zhang

Xinping Zhang

Yuqing Zhang

Zijun Zhang

Chunfeng Zhao

Kristin Zhao

Guoyan Zheng

Li Wu Zheng

Bashir Zikria

Werner Zimmerli

Michael Zindrick

Lewis Zionts

Israel Ziv

Joseph Zuckerman

Jozef Zustin

Gregory Zych

Michael Zywiel
Singapore, Singapore

Athens, Greece

Mersin, Turkey

Tianjin, China

Ami, Japan

Rochester, MN

Newcastle upon Tyne, United Kingdom

North Shore City, New Zealand

Vancouver, Canada

Rochester, MN

Hatyai, Thailand

Cleveland, $\mathrm{OH}$

Danville, $P A$

Los Angeles, $C A$

Santa Monica, CA

San Antonio, TX

San Francisco, $C A$

Nottingham, United Kingdom

Rochester, $N Y$

Boston, MA

Baltimore, $M D$

Rochester, MN

Rochester, MN

Bern, Switzerland

Hong Kong, China

Baltimore, $M D$

Liestal, Switzerland

Hinsdale, IL

Los Angeles, CA

Buffalo, NY

New York, $N Y$

Münster, Germany

Miami, FL

Toronto, Canada 